\title{
Analyse de la variabilité spatio-temporelle de l'îlot de chaleur urbain à Strasbourg (France)
}

\author{
Nathalia Philipps * Pierre P. Kastendeuch et Georges Najjar \\ Laboratoire Icube (UMR 7357) - Université de Strasbourg, 67412 Illkirch Cedex, France
}

\begin{abstract}
Résumé - Une analyse de la dynamique temporelle et de la distribution spatiale de l'îlot de chaleur urbain (ICU) strasbourgeois a été menée à l'aide d'un réseau de stations météorologiques réparties sur l'ensemble du territoire de l'agglomération strasbourgeoise. L'importante variabilité temporelle de l'ICU est illustrée non seulement à travers son comportement thermique journalier, mais également par le biais des fortes différences d'intensité selon les saisons et les types de temps. Favorisé lors de vents faibles et d'ensoleillement important, l'ICU se montre particulièrement intense durant les belles journées estivales, les moyennes pouvant localement atteindre un gain de cinq degrés lors du paroxysme nocturne. Concernant l'aspect spatial, les disparités entre stations soulignent une hétérogénéité de l'ICU essentiellement liée à la variabilité intrinsèque du milieu urbain. L'analyse statistique a ainsi mis en évidence le rôle de plusieurs paramètres morphologiques et d'occupation du sol, et par conséquent justifie pleinement la mise en place d'une classification en Local Climate Zones (LCZ) de l'Eurométropole de Strasbourg. La végétation apparaît comme étant un facteur de mitigation prééminent, notamment lorsqu'elle est présente de manière notable dans des zones densément bâties et fortement minéralisées. Concernant les paramètres relevant de la géométrie urbaine, les intensités moyennes d'ICU les plus élevées sont systématiquement mesurées dans les zones les plus densément bâties. Une nouvelle méthodologie de cartographie de l'ICU se basant sur les paramètres sous-jacents des LCZ est proposée. Cette carte permet l'obtention de valeurs pertinentes d'intensité de l'ICU en tout point du territoire.
\end{abstract}

Mots-clés : îlot de chaleur urbain / $\mathrm{LCZ}$ / végétation / géométrie urbaine / $\Delta \mathrm{T}_{\mathrm{ur}}$

\begin{abstract}
Spatio-temporal analysis of the urban heat island (UHI) in Strasbourg, France. The temporal dynamics and the spatial distribution of the urban heat island (UHI) in Strasbourg were analysed by using a substantial weather stations network which are distributed over the territory. The strong temporal variability of the phenomenon is illustrated both with regard to its diurnal variations and significant intensity differences according to seasons and weather conditions types. Most developed under cloudless and calm conditions, the UHI shows high magnitudes during sunny summer days, with means locally reaching up to $5^{\circ} \mathrm{C}$ at nocturnal peak. Regarding the spatial aspect, the intensity variations between the stations highlight the heterogeneity of the UHI, which is primarily explained by the diversity of urban forms. The statistical analysis has thus permitted to underscore the role of several morphological and land cover parameters, justifying the use of a Local Climate Zones (LCZ) classification of the Eurométropole of Strasbourg (EMS). The vegetation seems to have a predominant mitigation potential, in particular when it is notably planted in high-mineral, densely builtup areas. The strongest mean magnitudes are actually systematically measured in most built-up urban areas. This paper proposes a new mapping method for the spatialization of the UHI, which depends partly on LCZ parameters. The map presents relevant intensity values all over the metropolitan area.
\end{abstract}

Keywords: urban heat island / LCZ / vegetation / urban geometry / $\Delta \mathrm{T}_{\mathrm{ur}}$

* Auteure de correspondance $:$ nathalia.philipps@etu.unistra.fr

\section{Introduction}

Dans un contexte de réchauffement climatique global, il est fort probable que les vagues de chaleur seront amenées à être plus fréquentes, plus longues et plus intenses. D'ici la seconde moitié du $21^{\text {ème }}$ siècle, les températures relevées lors de la canicule de 2003 pourraient ainsi devenir la nouvelle norme 
estivale (De Munck et al., 2013). Les effets néfastes des vagues de chaleur seront davantage ressentis en ville, au sein desquelles le phénomène d'îlot de chaleur urbain (ICU) tend à exacerber le stress thermique engendré, entraînant de graves conséquences sanitaires pour les citadins (Arnfield et al., 2003 ; Zhao et al., 2018).

La mise en place d'un ICU résulte en premier lieu de modifications de l'occupation du sol par rapport à la campagne environnante. Morphologie urbaine, propriétés thermiques des matériaux de construction et flux de chaleur anthropique sont autant d'aspects entraînant une hausse des températures en milieu urbain (Oke, 1995). Diverses stratégies de mitigation tentant d'agir sur ces facteurs sont actuellement mises en place par les villes, dans le cadre de politiques d'adaptation au changement climatique. La plupart sont centrées sur l'introduction de végétation, dont les effets d'atténuation ont déjà été l'objet de nombreuses publications (Spronken-Smith et Oke, 1998 ; Bowler et al., 2010). Cependant les facteurs liés à la géométrie urbaine jouent également un rôle crucial dans l'établissement d'un ICU (Andreou, 2014), les rendant indissociables d'une politique de mitigation efficace.

Face à l'hétérogénéité du milieu urbain, il apparaît alors essentiel de mieux cerner l'influence de ces différentes variables sur le comportement de l'ICU. Visant à l'origine la comparaison entre études d'ICU de différentes villes à travers le monde, les Local Climate Zones (LCZ) (Stewart et Oke, 2012) peuvent également en améliorer la compréhension à l'échelle intra-urbaine. Les 17 types de LCZ existants reposent en effet sur une dizaine de caractéristiques relevant aussi bien de l'occupation du sol que de la morphologie urbaine, et permettent ainsi de prédire l'intensité de l'ICU au sein d'une zone donnée, tout en soulignant le rôle de chaque paramètre dans la mise en place du processus. Or si des cartographies LCZ ont été mises en place pour diverses villes, peu d'études ont encore directement employé les valeurs des paramètres composant les LCZ dans l'objectif de cartographier l'ICU sur un territoire donné.

Cette étude se propose de fournir une analyse statistique de la variabilité de l'ICU strasbourgeois, portant aussi bien sur sa dynamique temporelle que sur sa distribution spatiale. Elle vise à obtenir un aperçu de son intensité au sein d'un type de zone urbaine donné, et ainsi à mettre en évidence le rôle de plusieurs variables géométriques et d'occupation $\mathrm{du}$ sol dans une optique d'amélioration des stratégies de mitigation existantes. L'analyse se conclut par une nouvelle méthodologie de cartographie de l'ICU s'appuyant sur les paramètres sous-jacents des LCZ et les travaux d'Unger (2006).

\section{Site d'étude, matériel et méthodes}

\subsection{Présentation de la zone d'étude et des sites de mesures}

Agglomération composée de 33 communes, l'Eurométropole de Strasbourg (EMS) occupe une superficie de $340 \mathrm{~km}^{2}$ et regroupe plus de 491000 habitants (chiffres INSEE 2016). Localisée dans le nord-est de la France $\left(48,583^{\circ} \mathrm{N}-7,745^{\circ} \mathrm{E}\right.$; figure 1a), elle présente un climat tempéré de type $\mathrm{Cfb}$ dégradé d'après la classification actualisée de Köppen-Geiger. La température moyenne y est de $10,9^{\circ} \mathrm{C}$, avec un minimum et un maximum mensuels moyens respectifs de $1,8^{\circ} \mathrm{C}$ en janvier et $20,1^{\circ} \mathrm{C}$ en juillet (normales climatiques 19812010, Météo France). Les précipitations annuelles cumulées atteignent $665 \mathrm{~mm}$, le minimum de 32,2 mm se présentant en janvier et le maximum de 81,9 $\mathrm{mm}$ en mai. En outre, l'EMS bénéficie d'un contexte topographique la prédisposant aux études de climatologie urbaine, avec un très faible dénivelé (10 $\mathrm{m}$ au maximum) et une situation quasi-plane.

L'agglomération dispose d'un réseau de plus d'une trentaine de stations météorologiques déployées sur l'ensemble de son territoire (figure 1b). Trois organismes se partagent leur gestion : Météo France, ATMO Grand Est et le laboratoire Icube. Celles dépendant des deux premières structures sont des stations permanentes, tandis que les autres ont été installées durant une campagne de mesures menée par l'équipe TRIO d'Icube entre 2013 et 2016, dans le cadre d'un projet de recherche visant à comprendre et modéliser la réponse de la zone urbaine strasbourgeoise aux conditions climatiques imposées (Najjar et al., 2015). 


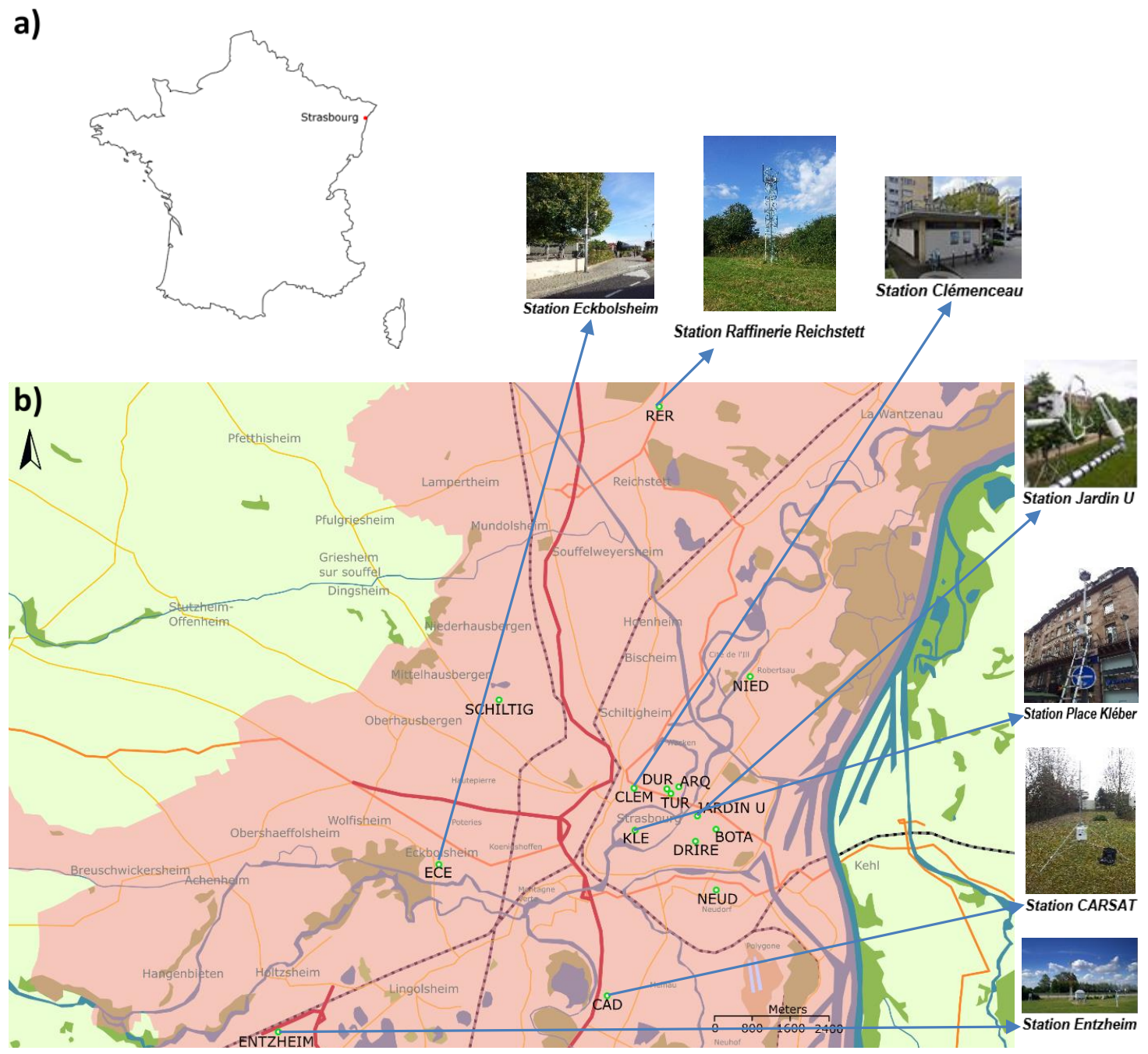

Figure 1. a) Localisation de la ville de Strasbourg en France. b) Carte des 15 stations utilisées (sources des données : réseaux ICube, ATMO Grand Est et Météo France. Sources des photos : Google Map, Colin J pour ICube, Strasbourg). a) Location of Strasbourg city in France. b) Map of the 15 used weather stations.

Les stations occupent des zones aux caractéristiques géométriques et d'occupation du sol variées. Certaines sont ainsi associées à un milieu urbain extrêmement minéral (stations situées au centre-ville comme la station Kléber), tandis que d'autres se situent au niveau de parcs urbains (stations du Jardin Botanique et du Jardin du Palais U) ou au sein du milieu rural (station d'Entzheim).

\subsection{Matériel et méthodes}

Afin d'obtenir une robustesse statistique la plus conséquente possible, seules cinq stations permanentes disposant d'une période de mesures étendue ont été sélectionnées pour la première partie de l'étude. Les données horaires de température de l'air $\left({ }^{\circ} \mathrm{C}\right)$ recouvrent ainsi sept années pleines (2013-2019), et sont mesurées à 1,5 $\mathrm{m}$ au-dessus du sol avec des capteurs électriques à résistance de platine fonctionnant avec la précision requise pour les standards météorologiques. Installée sur un toit d'un bâtiment à $20 \mathrm{~m}$ de hauteur, la DRIRE est la seule à déroger à la règle. Or les données de ce site ont été comparées avec celles d'une autre station dotée d'une période de mesures bien moins longue, aux caractéristiques similaires mais installée au niveau de la chaussée, et ont présenté un comportement proche avec un coefficient de corrélation (r) de 0,999 et une erreurtype (ET) de $0,367^{\circ} \mathrm{C}$ (Kastendeuch et al., 2019).

L'intensité de l'ICU ( $\left.\Delta \mathrm{T}_{\mathrm{ur}}\right)$ à un instant t est obtenue en soustrayant la température de la station urbaine à celle de la station rurale de référence. Les 
écarts horaires de température sont ainsi systématiquement calculés entre une station $\mathrm{x}$ et celle d'Entzheim, donnant l'intensité de l'ICU $\left({ }^{\circ} \mathrm{C}\right)$ mesurée au niveau de la première :

$$
\Delta \operatorname{Tur}_{(t)}=T_{\text {Station } x(t)}-T_{\text {Station Entzheim }(t)}
$$

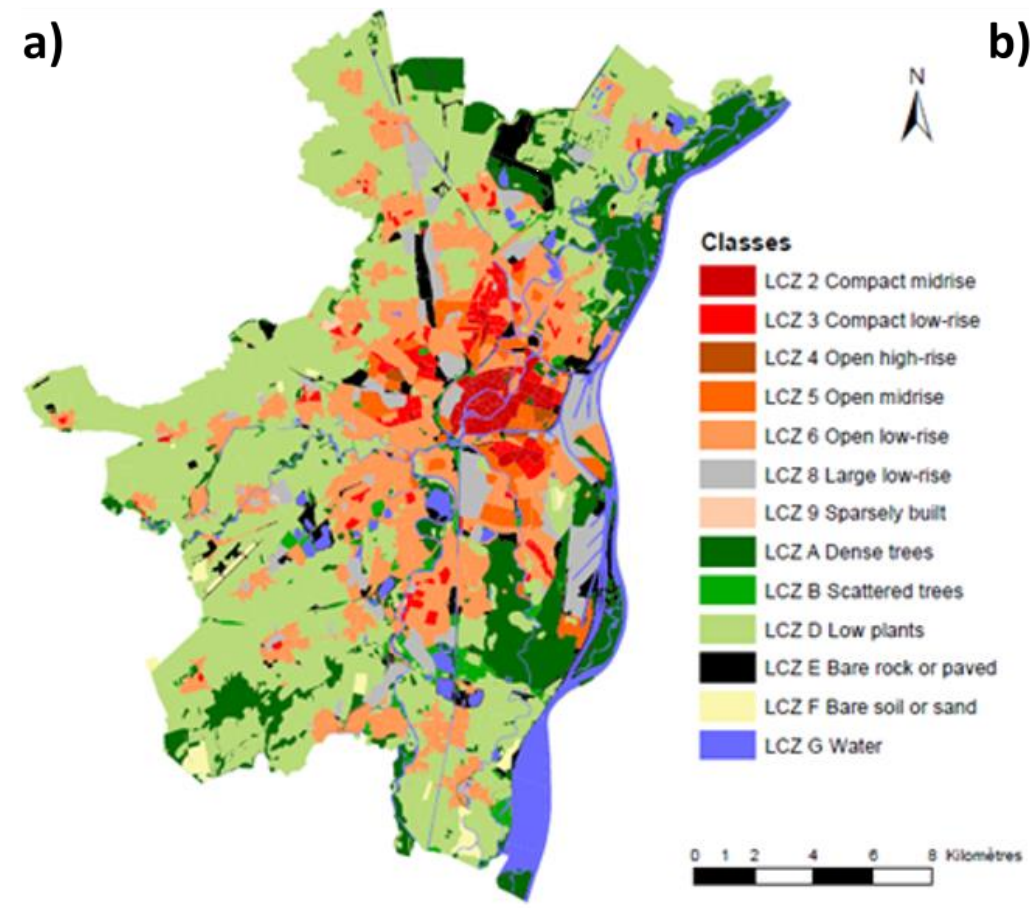

\begin{tabular}{|c|c|}
\hline $\begin{array}{l}\text { Paramètres originels de } \\
\text { Stewart et Oke (2012) }\end{array}$ & $\begin{array}{c}\text { Paramètres retenus } \\
\text { dans la classification } \\
\text { LCZ de l'EMS }\end{array}$ \\
\hline Sky View Factor (SVF) & Sky View Factor (SVF) \\
\hline Aspect ratio & / \\
\hline $\begin{array}{c}\text { Building Surface Fraction } \\
\text { (BSF) }\end{array}$ & $\begin{array}{l}\text { Building Surface } \\
\text { Fraction (BSF) }\end{array}$ \\
\hline $\begin{array}{c}\text { Impervious Surface } \\
\text { Fraction (ISF) }\end{array}$ & $\begin{array}{c}\text { Impervious Surface } \\
\text { Fraction (ISF) }\end{array}$ \\
\hline $\begin{array}{l}\text { Pervious Surface } \\
\text { Fraction (PSF) }\end{array}$ & $\begin{array}{l}\text { Pervious Surface } \\
\text { Fraction (PSF) }\end{array}$ \\
\hline $\begin{array}{l}\text { Height of roughness } \\
\text { elements }\end{array}$ & $\begin{array}{l}\text { Height of roughness } \\
\text { elements }\end{array}$ \\
\hline Surface albedo & Surface albedo \\
\hline Terrain roughness class & / \\
\hline Surface admittance & / \\
\hline $\begin{array}{l}\text { Anthropogenic heat } \\
\text { output }\end{array}$ & / \\
\hline / & $\begin{array}{l}\text { Vegetation Surface } \\
\text { Fraction }(\text { VgSF) }\end{array}$ \\
\hline
\end{tabular}

Figure 2. a) Cartographie LCZ de l'EMS (Montauban, 2019). b) Paramètres employés pour la classification LCZ de I'EMS comparés à ceux définis par Stewart \& Oke (2012). a) LCZ map of EMS (Montauban, 2019). b) Comparison between used properties for LCZ classification of EMS and those defined in Stewart \& Oke (2012).

La figure 2a présente la classification LCZ de l'EMS obtenue par Montauban (2019) d'après Stewart et Oke (2012). Outre six des dix paramètres définissant chaque LCZ (figure $2 b$ ), il a été choisi d'y ajouter le pourcentage de surface végétalisée (VgSF), permettant ainsi d'opérer une distinction supplémentaire entre stations appartenant à un même type de LCZ et de mettre en évidence le rôle de la végétation dans la spatialisation de l'ICU. Celle-ci est illustrée à travers une cartographie basée sur la mise en place d'une régression linéaire multiple (MLR) entre l'intensité maximale moyenne de l'ICU ( $\Delta \mathrm{T}_{\text {ur }} \max$ moyen) et les paramètres LCZ.

Contrairement à l'aspect temporel de l'ICU analysé via l'étude de quatre couples sur sept ans, la mise au point de cette cartographie repose sur dixsept stations urbaines étudiées sur une période d'une vingtaine de jours de l'année 2015. Afin d'obtenir un $\Delta \mathrm{T}_{\text {ur }}$ max moyen en tout point de l'EMS, plusieurs équations de MLR sont calculées avec le logiciel SAGA GIS à l'aide des valeurs des paramètres LCZ de chaque station. Leur qualité variant fortement en fonction des paramètres introduits en prédicteurs, seule la MLR au coefficient de détermination le plus élevé $(\mathrm{R} 2=$ $0,71)$ a été retenue. Le $\Delta \mathrm{T}_{\text {ur }} \max$ moyen calculé pour cette étude dépend ainsi uniquement du BSF (fraction de surface bâtie), de l'ISF (fraction de surface imperméable) et du VgSF. Il est alors possible d'en calculer une valeur théorique pour chaque polygone LCZ de 1'EMS en appliquant l'équation de régression. Un lissage des résultats obtenus est effectué via une moyenne mobile afin d'éviter les transitions trop abruptes entre deux LCZ voisines.

\section{Variabilité temporelle de I'ICU strasbourgeois}

De nombreuses études concernant des villes issues de zones climatiques très différentes ont déjà 
mis en évidence le cycle journalier de l'ICU et sa prédominance nocturne. Or chaque cité dispose d'une combinaison unique de facteurs climatiques, morphologiques et géographiques pouvant occasionner quelques différences au niveau de la survenue de l'intensité maximale ou minimale horaire du phénomène (Eastin et al., 2018). Quatre stations, en plus de la station rurale d'Entzheim (Météo France, LCZ B), illustrent la variabilité temporelle de l'ICU strasbourgeois sur la période 2013-2019 : DRIRE (ATMO Grand Est, LCZ 2), Botanique (Météo France, LCZ 2 végétalisée), Neudorf (ATMO Grand Est, LCZ 3) et Schiltigheim (ATMO Grand Est, LCZ 6). Cette variabilité est analysée à travers son évolution horaire journalière, puis du point de vue saisonnier avant d'être liée aux différents types de temps.

\subsection{Dynamique moyenne journalière et saisonnière}

L'opposition entre comportement diurne et nocturne de l'ICU apparaît clairement à travers les courbes du $\Delta \mathrm{T}_{\text {ur }}$ horaire moyen de la figure 3a : l'intensité maximale moyenne est atteinte aux alentours de minuit pour toutes les stations, quel que soit leur type de LCZ. Le processus s'amplifie après le coucher du soleil, moment à partir duquel la zone rurale se refroidit nettement plus vite que le milieu urbain, et décline progressivement lors du lever du jour. En période diurne, le milieu rural tend à se réchauffer plus vite du fait d'un ombrage réduit et de l'importante inertie thermique des matériaux des zones urbaines, réduisant significativement les écarts de température entre ville et campagne. Cela peut même conduire à la survenue d'un îlot de fraîcheur urbain (IFU), y compris pour des zones très minéralisées en centre-ville. Un premier aperçu de l'hétérogénéité spatiale de l'ICU est souligné ici, avec une intensité nocturne en moyenne plus importante pour les stations au caractère urbain prononcé.

Ce cycle journalier en apparence régulier ne doit pas masquer l'importante variabilité de l'heure d'apparition ou de disparition de l'ICU (figure $3 b$ ) : celui-ci peut en effet survenir à n'importe quel moment de la journée, voire même atteindre son maximum en plein jour (Najjar, 2006). D'autres aspects sont à prendre en compte, notamment le facteur saisonnier.

L'hétérogénéité saisonnière mesurée au niveau de Neudorf (figure 3c) révèle des courbes ayant une forme similaire à celle du $\Delta \mathrm{T}_{\mathrm{ur}}$ horaire moyen annuel, les principales différences concernant essentiellement l'amplitude du phénomène. Le printemps et l'automne enregistrent ainsi un ICU maximal moyen similaire, de $1^{\prime}$ ordre de $1,9^{\circ} \mathrm{C}$. $\mathrm{La}$ saison vernale présente également un léger IFU matinal $\left(-0,1^{\circ} \mathrm{C}\right)$. Apparaissant comme une exacerbation des saisons d'équinoxes, l'été se caractérise à la fois par l'ICU maximal moyen le plus intense $\left(\sim 2,6^{\circ} \mathrm{C}\right)$ et un IFU moyen plus long et plus prononcé $\left(\sim-0,3^{\circ} \mathrm{C}\right)$ que son pendant printanier. La courbe du $\Delta \mathrm{T}_{\text {ur }}$ horaire moyen hivernal est la seule à présenter une forme différente. La relative homogénéité des valeurs (moins de $1^{\circ} \mathrm{C}$ de différence entre le maximum et le minimum), ainsi que la persistance d'un ICU matinal, peuvent résulter de deux phénomènes : la hausse saisonnière des émissions de chaleur anthropique provoquée par le regain d'utilisation du chauffage, et la présence notable de conditions météorologiques défavorables à l'installation d'un ICU. Or ni l'intensité, ni l'heure d'apparition ou de disparition du processus (figure $3 \mathrm{~d}$ ) ne peuvent être expliquées par le seul facteur saisonnier. Le fait que les $\Delta \mathrm{T}_{\text {ur }}$ moyens les plus élevés se produisent davantage durant les nuits estivales, période la plus propice aux belles journées, souligne surtout l'importance du lien entre type du temps et intensité de l'ICU.

\subsection{Variabilité de I'ICU en fonction des conditions météorologiques}

De nombreuses études ont déjà mis en évidence le rôle de la nébulosité et de la vitesse du vent sur l'ICU moyen (Cantat, 2004). Quatre types de temps sont abordés ici afin de démontrer l'influence prépondérante de ces deux paramètres sur son intensité (figure 4a). Nous avons choisi de définir les jours de beau temps, favorables au développement d'un ICU, comme étant caractérisés par une fraction d'insolation (FIN) supérieure ou égale à $80 \%$ et une vitesse moyenne de vent inférieure ou égale à 3 m.s- 1 . 
a)

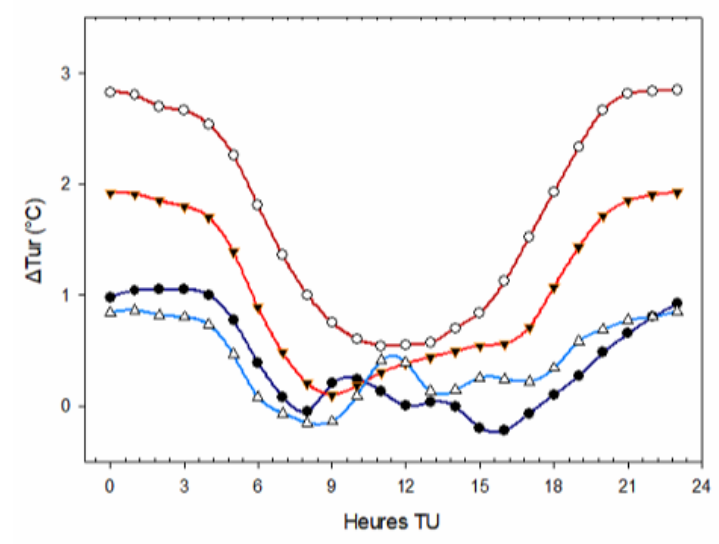

\begin{tabular}{l}
$\rightarrow-\infty$ DTur BOTA-ENTZ (LCZ 2 végétalisée) \\
\hdashline- DTur DRIRE-ENTZ (LCZ 2)
\end{tabular}

c)

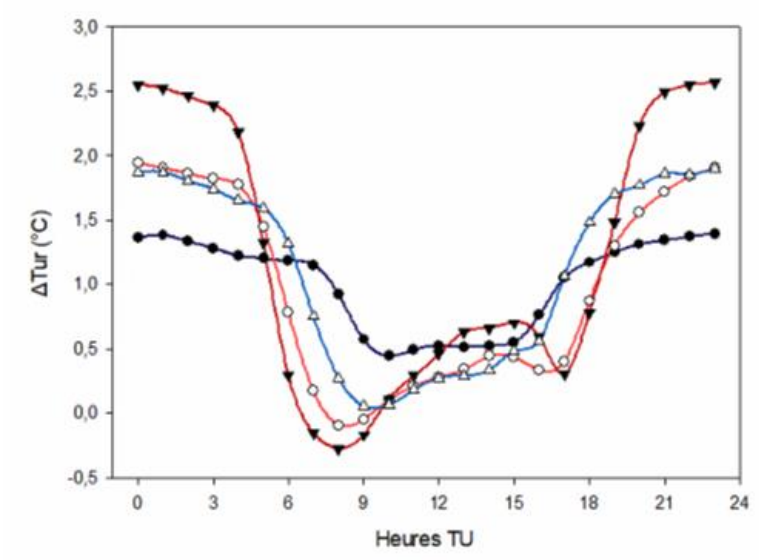

b)

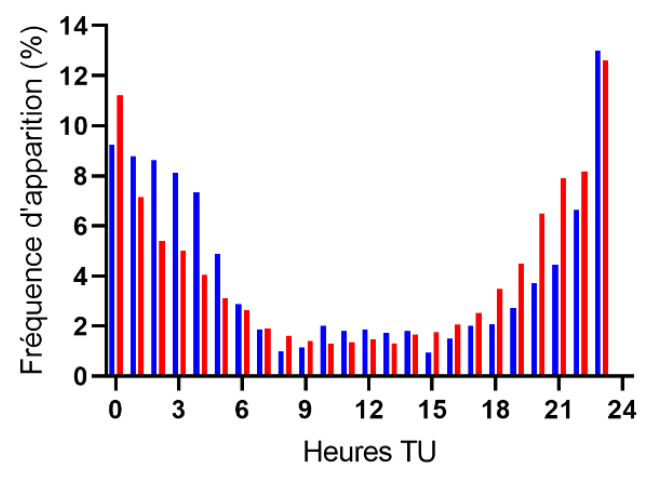

- Botanique-Entzheim DRIRE-Entzheim

d)

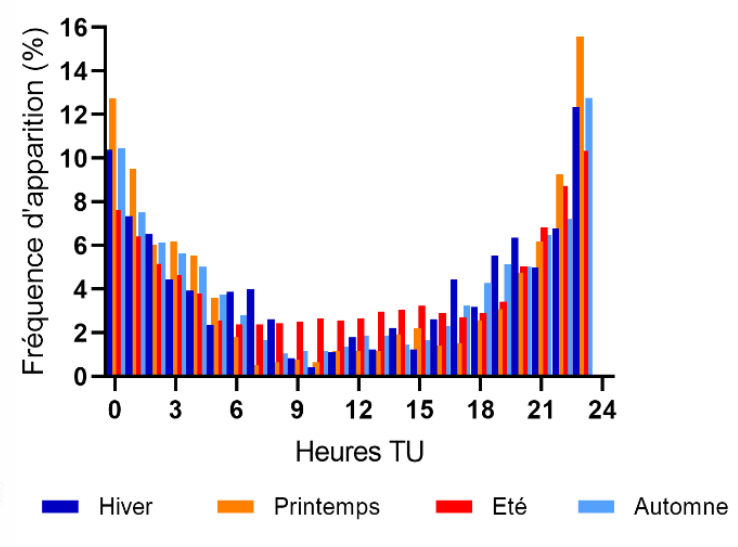

$\bullet-$ Hiner $\rightarrow-$ Printemps $\neg$ Eté $\rightarrow$ Autome

Figure 3. a) Intensité moyenne de l'ICU en fonction de l'heure. b) Fréquence d'apparition horaire du $\Delta$ Tur max pour Botanique et DRIRE. c) Moyennes saisonnières de l'intensité horaire de l'ICU pour Neudorf-Entzheim. d) Fréquence d'apparition du $\Delta$ Tur max en fonction de l'heure et la saison pour Neudorf-Entzheim. a) Mean hourly UHI intensity. b) Hourly frequency of $\Delta$ Tur max for Botanique and DRIRE stations. c) Seasonal hourly means of UHI intensity for NeudorfEntzheim. d) Hourly and seasonal frequencies of $\Delta$ Tur max for Neudorf-Entzheim.

L'ICU maximal moyen diminue en même temps que la FIN, en particulier pour les stations à caractère urbain marqué. Le $\Delta \mathrm{T}_{\text {ur }}$ max moyen de la DRIRE passe en effet d'une valeur de près de $6^{\circ} \mathrm{C}$ lors de belles journées à $2,81^{\circ} \mathrm{C}$ sous un ensoleillement absent. A l'opposé, seule une différence de $1,12^{\circ} \mathrm{C}$ sépare ces deux valeurs pour Botanique. La vitesse journalière moyenne du vent exerce également un rôle considérable dans la mitigation de l'ICU puisqu'à ensoleillement égal, elle permet lorsqu'elle est assez forte une diminution notable du $\Delta \mathrm{T}_{\text {ur }}$ max moyen (plus de $1,6^{\circ} \mathrm{C}$ pour la DRIRE). Lors d'une succession de journées de beau temps (figure 4b), ce paramètre apparaît même comme étant un facteur limitant la dissipation de l'ICU : outre un effet d'accumulation visible entraînant une augmentation progressive du $\Delta \mathrm{T}_{\text {ur }}$ max durant la période du 22 au 27 août 2019 (passant de $4,3^{\circ} \mathrm{C}$ à plus de $6,5^{\circ} \mathrm{C}$ les deux derniers jours), il subsiste un fort ICU lors de la journée du 28 août, présentant pourtant une FIN relativement basse $(30,5 \%)$. Or la faible vitesse de vent relevée $(1,47 \mathrm{~m} / \mathrm{s})$ semble avoir permis au phénomène de persister malgré l'occurrence d'un type de temps a priori défavorable à son maintien. 
a)

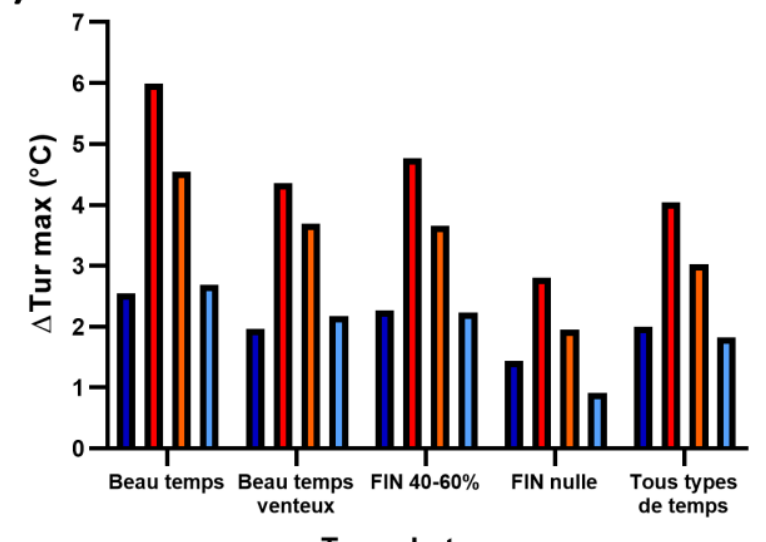

Type de temps b)

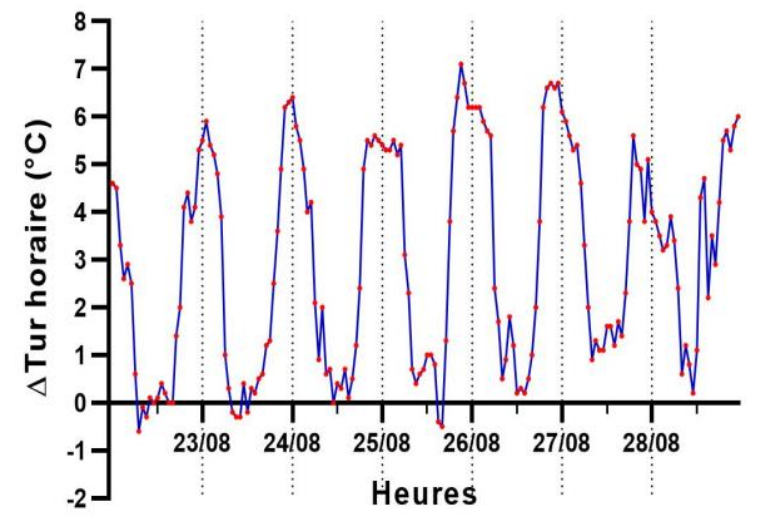

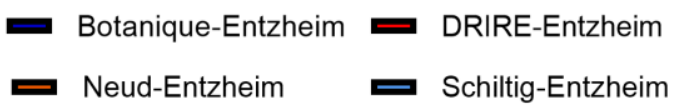

Figure 4. a) Intensité maximale journalière moyenne de l'ICU en fonction des types de temps sur la période 2013-2019. b) Evolution du $\Delta$ Tur sur une période de six jours de beau temps successifs pour DRIRE-Entzheim. a) Daily mean maximum UHI intensity according to the weather types during 2013-2019 period. b) Evolution of $\Delta$ Tur during a succession of six calm and clear weather days for DRIRE-Entzheim.

Une succession de jours de beau temps peut ainsi entraîner la persistance d'un fort ICU parfois plusieurs jours après son achèvement. L'augmentation de la fréquence et de la durée des vagues de chaleur, propices à ce type de journées, présente alors un risque accru d'exacerbation du $\Delta \mathrm{T}_{\text {ur }}$ max journalier et d'augmentation de la survenue d'ICU atypiques. L'étude de la variabilité temporelle du phénomène souligne la présence d'une forte hétérogénéité spatiale nécessitant d'être analysée au mieux dans le cadre d'une stratégie de mitigation optimale.

\section{Distribution spatiale de I'ICU strasbourgeois et cartographie}

Concernant l'évaluation de l'intensité de l'ICU, les 17 types de LCZ existants sont supposés représenter chacun un comportement thermique donné. Or il a déjà été souligné l'existence d'une variabilité intrinsèque au sein d'une unique catégorie de LCZ (Leconte et al., 2015). Plutôt que de concevoir des sous-classes de LCZ risquant de diminuer la pertinence même de la classification, il a été choisi de mettre en évidence le rôle discriminant de certains paramètres dans la mise en place d'une variabilité intra-LCZ. La prise en compte de cette dernière, ainsi que la détermination de l'influence de plusieurs paramètres-clés, permettent l'obtention d'une spatialisation de l'ICU reposant entièrement sur les LCZ.

\subsection{Mise en évidence du rôle de la végétation et de la géométrie urbaine via le comportement thermique des LCZ}

Les différents paramètres morphologiques et d'occupation du sol composant les LCZ n'ont pas le même degré d'influence sur l'ICU résultant. Concernant le BSF (figure 5a), la corrélation positive avec le $\Delta \mathrm{T}_{\text {ur }}$ max moyen $(\mathrm{R} 2=0,66)$ est évidente et ne fait que confirmer les résultats de plusieurs études antérieures (Oke, 1981) ayant déjà illustré le lien entre exacerbation de l'ICU et densification urbaine. D'autres variables présentent en revanche des résultats plus mitigés, notamment le Sky View Factor (SVF) dont le rôle sur 1'ICU semble dépendre de la configuration géométrique dans laquelle la station concernée s'inscrit, ambiguïté déjà exposée par $\mathrm{Hu}$ et al. (2016). Enfin, le VgSF (figure 5b) souligne l'importance des interactions entre différents paramètres : lorsque les 18 stations sont prises en compte dans la régression linéaire, la corrélation entre végétation et $\Delta \mathrm{T}_{\text {ur }} \max$ moyen amoindri apparaît très faible $(\mathrm{R} 2=0,17)$. Or si l'on choisit d'ôter quatre stations particulières, le potentiel de mitigation de la végétation transparaît bien davantage $(\mathrm{R} 2=0,43)$. Il s'avère que ces 
stations ont soit un $\mathrm{VgSF}$ important mais une fraction de surface perméable (PSF) très faible doublée d'un BSF non négligeable, soit la situation inverse. Plus que le rôle distinct de chacun de ces paramètres, ce sont ainsi les différentes combinaisons qui vont exercer une influence plus ou moins forte sur l'ICU, justifiant pleinement le recours aux LCZ.

Le test de l'Anova confirme les tendances thermiques significativement distinctes des stations

a)
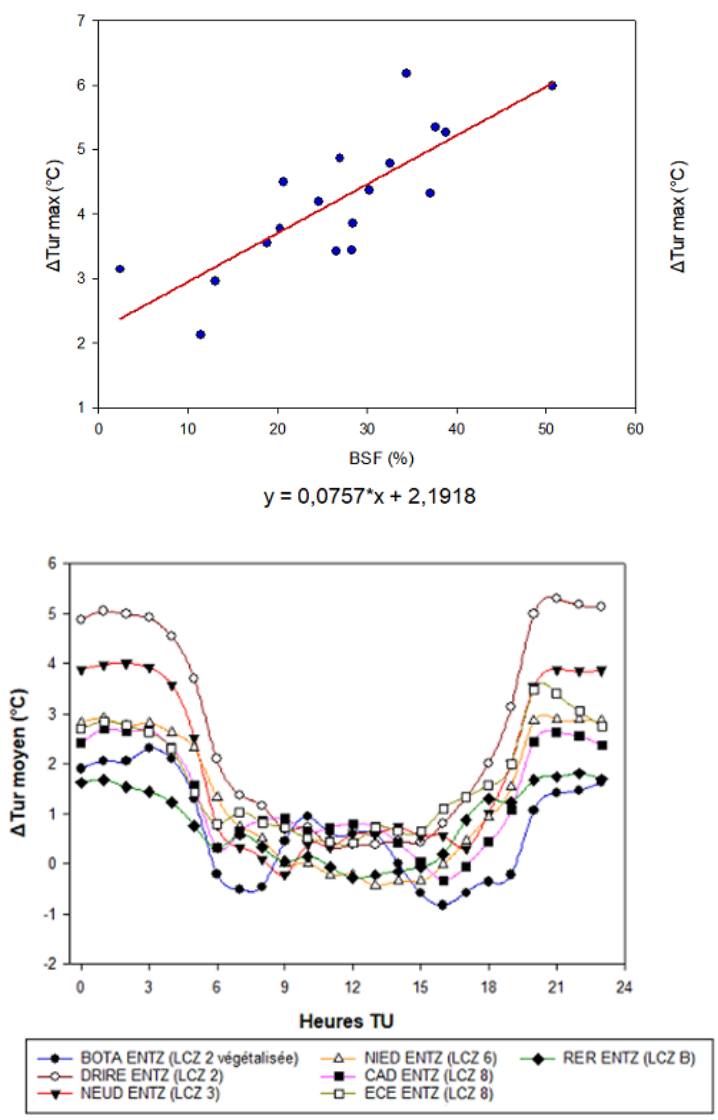

de la figure 5c, chacune associée à un type de LCZ donné. Les moyennes du $\Delta \mathrm{T}$ ur horaire corroborent sans surprise les intensités d'ICU plus élevées des LCZ les plus minéralisées et au bâti dense (types 2 et 3). Malgré son appartenance à une LCZ 2, Botanique se comporte davantage comme les stations CAD (LCZ 8) ou NIED (LCZ 6). La différence avec la DRIRE est hautement significative (plus de $4^{\circ} \mathrm{C}$ de différence aux alentours de $23 \mathrm{~h}$ TU) et illustre une importante variabilité intra-LCZ.

b)
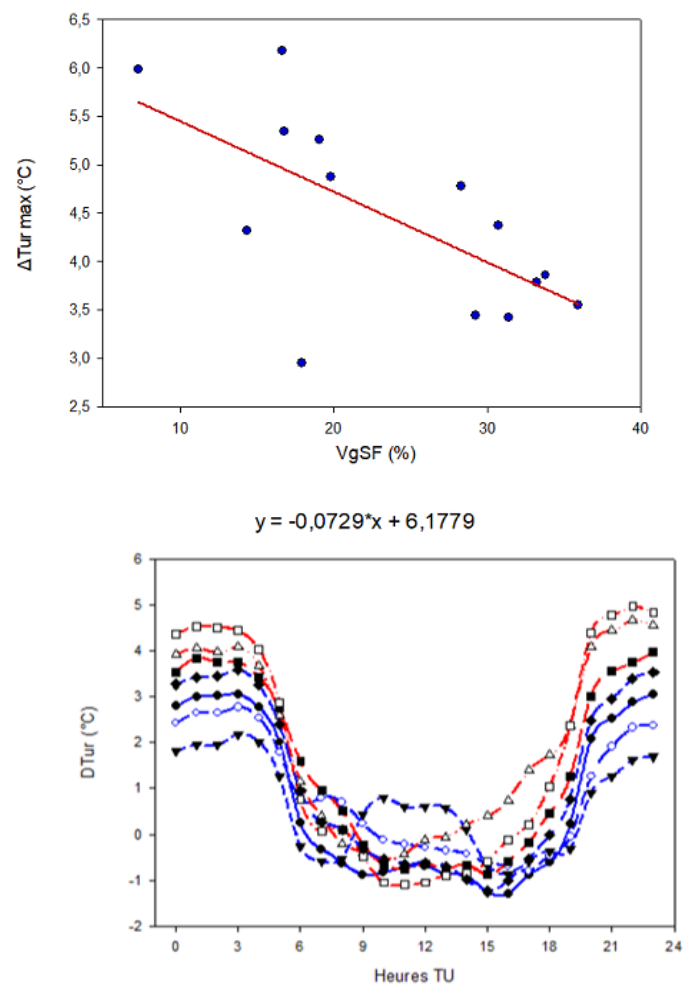

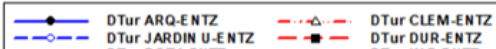
DTUR BOTAENTZ -1- DTUR BOTAENTE

Figure 5. a) Régression linéaire $\Delta$ Tur max - BSF. b) Régression linéaire $\Delta$ Tur max - VgSF. c) Intensité moyenne de I'ICU en fonction de l'heure sur la période de beau temps 2015 : variabilité inter-LCZ. d) Intensité moyenne de l'ICU en fonction de l'heure sur la période de beau temps 2015 : variabilité intra-LCZ pour les LCZ 2. a) Linear regression between $\triangle$ Tur max and BSF. b) Linear regression between $\triangle$ Tur max and VgSF. c) Mean hourly UHI intensity during the 2015 ideal weather period: inter-LCZ variability. d) Mean hourly UHI intensity during the 2015 ideal weather period: intra- $L C Z$ variability for $L C Z 2$.

La persistance d'une variabilité intra-LCZ est particulièrement mise en évidence au niveau des stations de l'étude appartenant au type 2 (figure 5d). Le potentiel de mitigation de la végétation apparaît très distinctement pour ce type de LCZ, de base propice à l'installation d'un ICU important. Or les quatre stations LCZ 2 les plus végétalisées ( $\mathrm{VgSF} \geq$ $29 \%$, en bleu) présentent non seulement des valeurs nocturnes d'ICU beaucoup plus faibles que celles l'étant moins (en rouge), mais aussi des changements concernant l'apparition et la durée du phénomène d'IFU. Celui-ci peut ainsi démarrer en 
milieu d'après-midi, représentant une possibilité d'atténuation du stress thermique non négligeable. $\mathrm{Ne}$ remettant nullement en cause la pertinence des LCZ, la variabilité intra-LCZ montre qu'en agissant sur certains paramètres (ici la végétation), il est possible de modifier le comportement thermique d'une zone urbaine et de mitiger localement l'ICU.

\subsection{Cartographie de I'ICU strasbourgeois}

Obtenue via la méthode décrite précédemment, la cartographie de l'ICU moyen maximum journalier (figure 6a) dépend entièrement de la qualité de la MLR et des valeurs prises par les paramètres LCZ sur l'ensemble du territoire. Les résultats permettent une distinction aisée des comportements thermiques de zones urbaines types comme le centre-ville, ainsi que la mise en évidence de certaines zones davantage périphériques mais présentant un ICU important. Or chaque polygone étant doté d'une unique valeur de $\Delta \mathrm{T}_{\text {ur }}$ max moyen, beaucoup de transitions très abruptes et irréalistes (pouvant aller jusqu'à $8^{\circ} \mathrm{C}$ de différence) subsistent au niveau des limites entre deux LCZ. Le lissage par moyenne mobile (figure 6b) atténue de manière notable cet effet. La pertinence de cette cartographie a été éprouvée via la comparaison du $\Delta \mathrm{T}_{\text {ur }} \max$ moyen mesuré d'une dix-huitième station urbaine, volontairement non-intégrée dans la MLR, avec celui prédit par la méthode. Le modèle cartographique diffère très peu de la mesure effectuée $\left(\Delta \mathrm{T}_{\text {ur }}\right.$ max cartographie $=4,14^{\circ} \mathrm{C} ; \Delta \mathrm{T}_{\text {ur }}$ max mesuré $=4,38)$. Ce type de cartographie constituerait de ce fait un outil potentiel efficace d'aide à la décision destiné aux urbanistes et aménageurs dans le cadre d'une volonté de mitigation de l'ICU.

Plusieurs limites persistent cependant pour cette première carte. Celle-ci repose ainsi sur une période d'une vingtaine de jours à peine, représentant un faible échantillon numérique et de ce fait une robustesse statistique amoindrie. Le fait qu'il s'agisse de la période recoupant le maximum de stations souligne également la nécessité de disposer d'un réseau météorologique le plus conséquent possible, et de préférence permanent, afin d'obtenir des MLR de qualité.

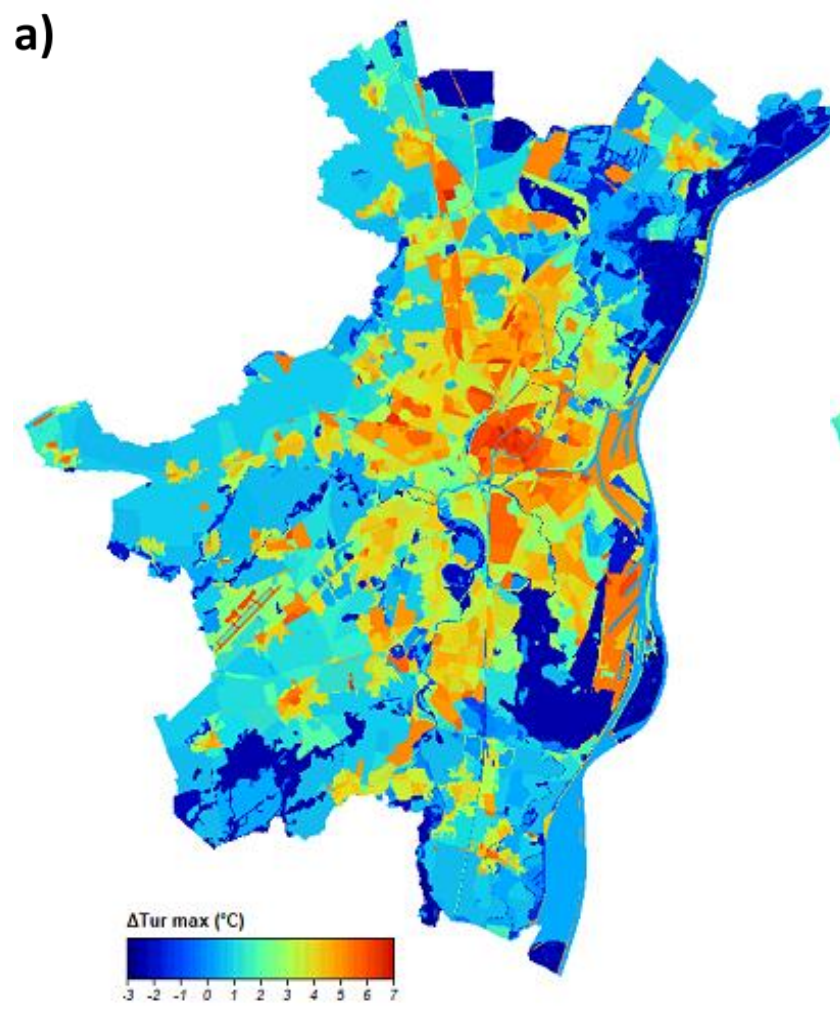

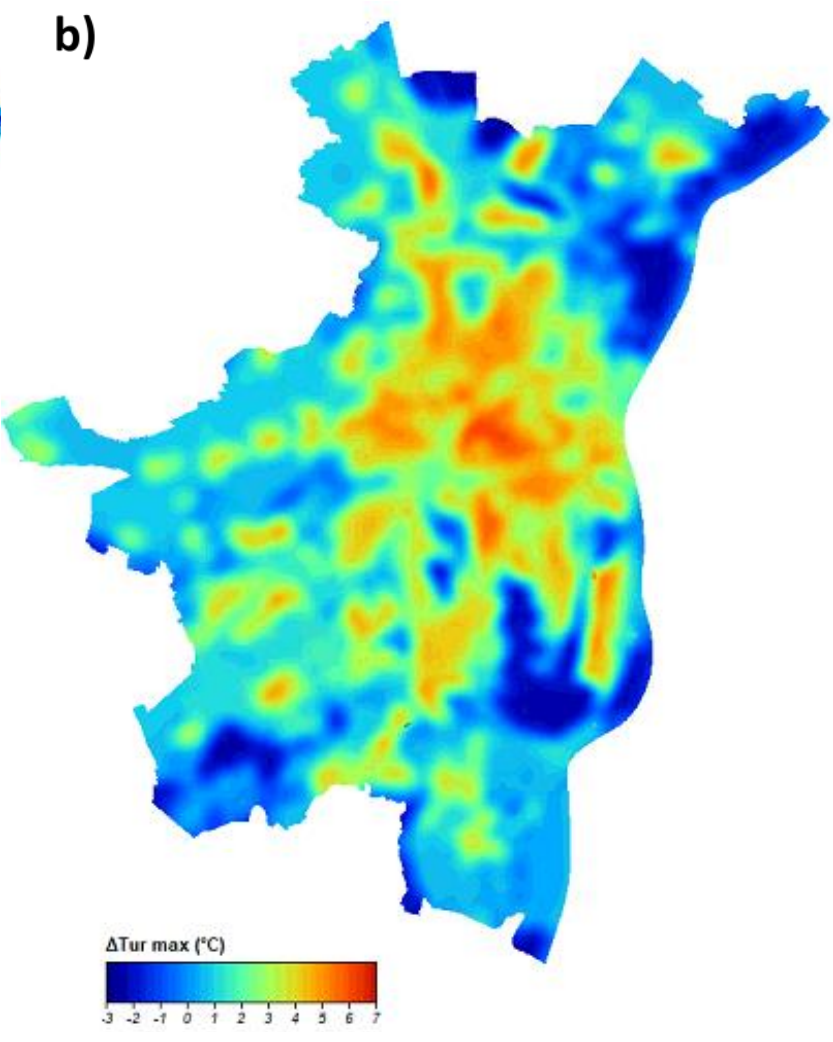

Figure 6. a) Cartographie originelle de l'ICU moyen maximum journalier, sans lissage. b) Cartographie de l'ICU moyen maximum journalier, avec lissage par moyenne mobile. a) First mean daily maximum UHI mapping, without smoothing. b) Mean daily maximum UHI mapping with smoothing by moving average. 


\section{Conclusion}

L'analyse de la variabilité temporelle de l'ICU strasbourgeois révèle que celui-ci connaît sa forme la plus intense lors des nuits calmes dénuées de nébulosité, à l'image de nombreuses autres villes à travers le monde. Malgré l'hétérogénéité saisonnière notable, les conditions météorologiques synoptiques restent le facteur temporel prépondérant dans sa mise en place et sa disparition. L'augmentation de la durée et de la fréquence des vagues de chaleur, couplée ainsi à une succession plus longue de journées propices au phénomène, augmentera fortement le risque de survenue d'un ICU particulièrement fort.

Dans l'étude de la distribution spatiale de l'ICU strasbourgeois, les LCZ se sont avérées être un outil fort pertinent, tant sur le volet statistique que sur la mise en place d'une cartographie à l'échelle d'un territoire métropolitain. Le potentiel de mitigation de la végétation, bien illustré à travers l'analyse d'une importante hétérogénéité au sein d'un type de LCZ, apparaît comme étant amélioré lorsque ce facteur s'inscrit dans une combinaison de paramètres favorable. Une atténuation optimale de l'ICU passera ainsi obligatoirement par une considération de l'ensemble des variables en jeu, notamment celles liées à la densité de bâti et à la perméabilité des sols, la végétation seule ne suffisant guère à compenser une morphologie propice au phénomène.

Cette cartographie de l'ICU peut être prometteuse, notamment en matière d'estimation de son intensité au niveau de zones dénuées de stations de mesures. Cependant, elle nécessite plusieurs améliorations, en particulier concernant le paramétrage du lissage par moyenne mobile, ainsi qu'une validation plus rigoureuse. Outre l'établissement de nouvelles cartographies de l'ICU, il serait alors possible d'étendre cette méthodologie à d'autres variables climatiques, voire aux indices de confort thermique.

\section{Références}

Andreou E., 2014. The effect of urban layout, street geometry and orientation on shading conditions in urban canyons in the Mediterranean. Renewable Energy, 63, 587-596.
Arnfield J. A., 2003. Two decades of urban climate research: a review of turbulence, exchanges of energy and water, and the urban heat island. International Journal of Climatology, 23 (1), 1-26.

Bowler D. E., Buyung-Ali L., Knight T. M., Pullin A. S., 2010. Urban greenings to cool towns and cities, a systematic review of the empirical evidence. Landscape and urban planning, 97 (3), 147-155.

Cantat O., 2004. L'îlot de chaleur urbain parisien selon les types de temps. Norois, 191.

De Munck C., Pigeon G., Masson V., Meunier F., Bousquet P., Tréméac B., Merchat M., Poeuf P., Marchadier C., 2013. How much air conditioning can increase air temperatures for a city like Paris? International Journal of Climatology, 33, 210-227.

Eastin M. D., Baber M., Boucher A., Di Bari S., Hubler R., Stimac-Spalding B., Winesett T., 2018. Temporal variability of the Charlotte (sub)urban heat island. Journal of Applied Meteorology and Climatology, 57, 81-102.

Hu Y., White M., Ding W., 2016. An urban form experiment on urban heat island effect in high density area. Procedia Engineering, 169, 166-174.

Kastendeuch P., Najjar G., Philipps N., 2019. Ilot de sécheresse et d'humidité à Strasbourg (France). Climatologie, 16, 72-90.

Leconte F., Bouyer J., Claverie R., Pétrissans M., 2015. Using Local Climate Zone scheme for UHI assessment: evaluation of the method using mobile measurements. Building and Environment, 83, 39-49.

Montauban O., 2019. Une approche vectorielle pour classifier des données images et vecteurs dans le but d'extraire une cartographie des Local Climate Zones des quartiers de Strasbourg. PFE, INSA Strasbourg.

Najjar G., 2006. Etudes méso-climatiques en milieu hétérogène : de la moyenne montagne à la ville. Habilitation à Diriger des Recherches, Université Louis Pasteur, Strasbourg I.

Najjar G., Colin J., Kastendeuch P., Ngao J., Saudreau M., Landes T., Améglio T., Luhahe R., Guillemin S., Schreiner G., Kleinpeter J., Nerry F., 2015. A three years long fieldwork experiment to monitor the role of vegetation on the urban climate of Strasbourg, France. 9th International Conference on Urban Climate, ICUC9, Toulouse, France, 6p

Oke T., 1981. Canyon Geometry and the Nocturnal Urban Heat Island: Comparison of Scale Model and Field Observations. Journal of Climatology, 1, 237-254.

Oke T., 1995. The heat island of the urban boundary layer: Characteristics, causes and effects. Nat. Adv. Sci. Inst. Ser., 277, 81-107.

Spronken-Smith R. A. et Oke T., 1998. The thermal regime of urban parks in two cities with different summer climates. International Journal of Remote Sensing, 19 (11), 2085-2104. Stewart I. D. et Oke T. R., 2012. Local Climate Zones for 
Urban Temperature Studies. Bulletin of the American Meteorological Society, 93, 1879-1900.

Unger J., 2006. Modelling of the annual mean maximum urban heat island using 2D and 3D surface parameters. Climate Research, 30 (3), 215-226.
Zhao L., Oppenheimer M., Qing Z., Baldwin J. W., Ebi K. L, Bou-Zeid E., Guan K., Liu X., 2018. Interactions between urban heat islands and heat waves. Environmental Research Letters, 13 (3).

Citation de l'article : Philipps N., Kastendeuch P. P. et Najjar G., 2020. Analyse de la variabilité spatio-temporelle de l'îlot de chaleur urbain à Strasbourg (France). Climatologie, 17, 10. 